\title{
Topology-Preserving General Operators in Arbitrary Binary Pictures
}

\author{
Kálmán Palágyi \\ Department of Image Processing and Computer Graphics, \\ University of Szeged, Hungary \\ palagyi@inf.u-szeged.hu
}

\begin{abstract}
A general operator may transform a binary picture by changing both black and white points. Sequential operators traverse the points of a picture, and consider a single point for possible alteration, while parallel operators can alter a set of points simultaneously. An orderindependent transition function yields the same sequential operator for arbitrary visiting orders. Two operators are called equivalent if they produce the same result for each input picture. A transition function is said to be equivalent if it specifies a pair of equivalent parallel and sequential operators. This paper establishes a necessary and sufficient condition for order-independent transition functions, a sufficient criterion for equivalent transition functions, and a sufficient condition for topologypreserving parallel general operators in arbitrary binary pictures.
\end{abstract}

\section{Introduction}

A binary picture on a digital space is a mapping that assigns a color of black or white to each point [12. A reduction (or reductive) operator transforms a binary picture only by changing some black points to white ones; an operator that never turns a black point into white is called an addition (or an augmentative operator); a general (or reductive-augmentative) operator may change both black and white points [5]).

Parallel operators can alter all points that satisfy their transition functions simultaneously, while sequential operators traverse the points of a picture, and may alter just the actually visited point. The author introduced the notions of equivalent reductions 14 that can be extended for general operators. Two general operators are said to be equivalent if they produce the same result for each input picture. Sequential operators with the same transition function may produce different results for different visiting orders (raster scans) of points. An order-independent transition function [15] produces the same result for arbitrary visiting orders. A transition function is called equivalent if it specifies a pair of equivalent parallel and sequential operators.

Various algorithms (e.g., thinning 4], shrinking [5], generation of skeleton by influence zones (SKIZ) [16, warping of binary images [2, or narrow band algorithm in level set methods [6]) are required to preserve topology. Topology

E. Bayro-Corrochano and E. Hancock (Eds.): CIARP 2014, LNCS 8827, pp. 22-29 2014.

(C) Springer International Publishing Switzerland 2014 
preservation is well-understood for reductions and additions [7/10,11]13], but it cannot be said of general operators.

In 14 the author gave a sufficient condition for equivalent reductions assuming $(8,4)$ pictures on the $(2 \mathrm{D})$ square grid. In this amplified work, we present a necessary and sufficient condition for order-independent transition functions, a sufficient criterion is given for transition functions that specify pairs of equivalent sequential and parallel operators, and a sufficient condition is established for topology-preserving parallel general operators. These results are valid in arbitrary binary pictures.

\section{Basic Notions and Results}

In this paper, we use the fundamental concepts of digital topology as reviewed by Kong and Rosenfeld [12].

Consider a pair of adjacency relations $(k, \bar{k})$ on a digital space $V[12$. A $(k, \bar{k})$ digital picture on $V$ is a quadruple $(V, k, \bar{k}, B)$, where each point in $B \subseteq V$ is called a black point; each point in $V \backslash B$ is said to be a white point; $k$-adjacency is used for black points; $\bar{k}$-adjacency is assigned to white points. A black component is a $k$-component of $B$, while a white component is a $\bar{k}$-component of $V \backslash B$.

It is assumed that any picture $(V, k, \bar{k}, B)$ contains finitely many black points, hence the hull of that picture denoted by $\mathcal{H}(B)$ also contains finitely many (black and white) points. Note that the hull of a picture sampled on an orthogonal grid is the bounding box of the set of black points. Throughout this paper, transition functions of the considered operators are evaluated only for the elements of $\mathcal{H}(B)$.

Sequential and parallel general operators are illustrated in Algorithms 1 and 2. A point $p$ is alterable, if $\mathcal{T}(p, Y)=$ true, where $Y$ denotes the set of black points in the (actual) picture, i.e., $Y=S B \subseteq B$ in sequential operators (see Algorithm 1), and $Y=B$ in the parallel case (see Algorithm 2).

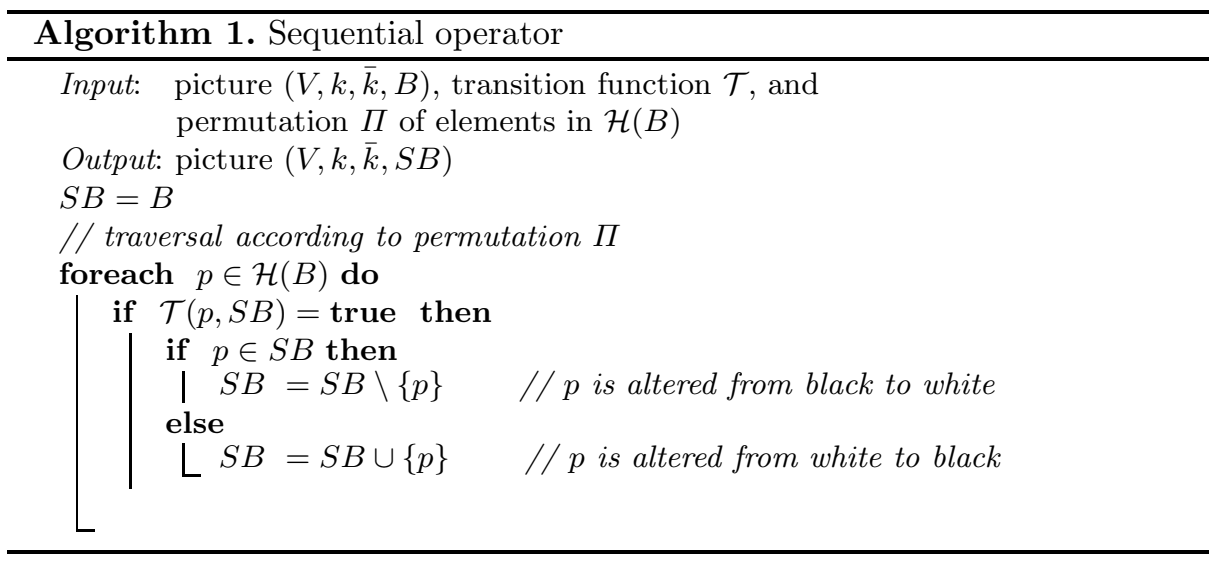




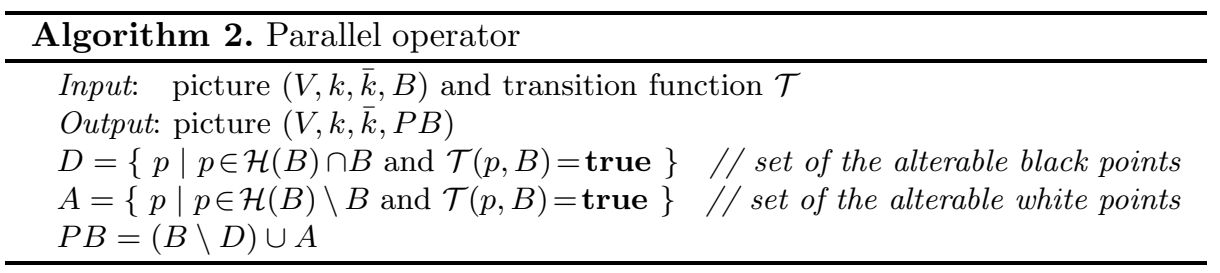

By comparing these two absolutely dissimilar approaches, we can state that in the parallel case (see Algorithm 2) the initial picture is considered when the transition function $\mathcal{T}$ is evaluated. On the contrary, the picture is dynamically altered when a sequential operation (see Algorithm 1) is performed. The transition function $\mathcal{T}$ is order-independent if the result of Algorithm 1 is uniquely specified by $\mathcal{T}$ (i.e., the resulted picture does not depend on the order $\Pi$ in which the points are selected by the foreach loop).

The support [4] of a transition function $\mathcal{T}$ applied at a point is a minimal set of points whose values determine whether the investigated points is altered by $\mathcal{T}$. Note that all topological algorithms uses local supports with "small" diameters. Let us denote by $\mathcal{S}_{\mathcal{T}}(p)$ the support of the transition function $\mathcal{T}$ with respect to a point $p$. It is easy to see that $\mathcal{T}(p, Y)=\mathcal{T}\left(p, Y \cap \mathcal{S}_{\mathcal{T}}(p)\right)$.

The concept of simple black points is well established in digital topology. A black point is simple in a picture if and only if its alteration is a topologypreserving reduction [12. There are several useful characterizations of simple points in 2D 11711,12, 3D [112, and 4D 111 pictures. This property can be extended for white points: a white point is simple in a picture if and only if its alteration is a topology-preserving addition.

Theorem 1. [5] A sequential operator is topology-preserving if its transition function alters only simple points.

Here we recall the following useful characterization of simple points on the (2D) square grid:

Theorem 2. [12] $A$ (black or white) point $p$ is simple in an $(8,4)$ picture if and only if $p$ is 4-adjacent to at least one white point, and the black points that are 8-adjacent to $p$ form exactly one 8-component.

A parallel general operator may alter a set of points and not just a single point. Hence it is needed to consider what is meant by topology preservation when a number of points are altered simultaneously. Various authors established sufficient conditions for parallel reductions to preserve topology 2D [7]10]13]14], 3D [10, and 4D 11] pictures. In 8] Kardos and Palágyi gave a sufficient condition for topology-preserving parallel general operators assuming $(8,4)$ pictures $[12$ on the (2D) square grid. 


\section{Criteria for Equivalent Transition Functions and Topology-Preserving Parallel Operators}

Let us define two special classes of transition functions.

Definition 1. Let $\mathcal{T}$ be a transition function, let $p \in \mathcal{H}(B)$ be a point in a picture $\mathcal{P}=(V, k, \bar{k}, B)$, let $q \in \mathcal{H}(B) \backslash\{p\}$ be a point that can be altered in picture $\mathcal{P}$ by $\mathcal{T}$. Transition function $\mathcal{T}$ is stable if the following conditions hold:

1. If $p$ can be altered in $\mathcal{P}$ by $\mathcal{T}$, then $p$ can be altered by $\mathcal{T}$ after the alteration of $q$.

2. If $p$ cannot be altered in $\mathcal{P}$ by $\mathcal{T}$, then $p$ cannot be altered by $\mathcal{T}$ after the alteration of $q$.

Definition 2. A transition function is simple-stable if it is stable, and it alters only simple points.

Let us state a necessary and sufficient condition for order-independent transition functions.

Theorem 3. A transition function is order-independent if and only if it is stable.

Proof. First let us prove the "if" part of this theorem.

Let us assume that Algorithm 1 with a transition function $\mathcal{T}$ produces the same result for any visiting orders of the set of points $\mathcal{H}(B)$ for any picture $\mathcal{P}=(V, k, \bar{k}, B)$. Let $p \in \mathcal{H}(B)$ a point in picture $\mathcal{P}$, let $q \in \mathcal{H}(B)$ be an alterable point in $\mathcal{P}$, and consider all possible permutations of the points in $\mathcal{H}(B)$. In some of these permutations, $p$ precedes $q$, and $q$ precedes $p$ in the remaining cases. Since $\mathcal{T}$ is order-independent, we get the same result for all possible permutations. Hence the alteration of $p$ does not depend on the value of $q$. Thus transition function $\mathcal{T}$ is stable.

Then we give an indirect proof of the "only if" part.

Let us assume that the Algorithm 1 with a stable transition function $\mathcal{T}$ produces different results for distinct visiting orders of the set of points $\mathcal{H}(B)$ with $n$ elements (i.e., $\mathcal{T}$ is not order-independent). It is obvious that $n \geq 2$ holds. All permutations of the elements of $\mathcal{H}(B)$ can be get by swapping a pair of successive points. (Recall the well-known bubble sort algorithm [9].) Let us consider the following two permutations

$$
\begin{aligned}
& \Pi_{1}(\mathcal{H}(B))=\left\langle x_{1}, \ldots, x_{i-1}, p, q, x_{i+2}, \ldots, x_{n}\right\rangle \text { and } \\
& \Pi_{2}(\mathcal{H}(B))=\left\langle x_{1}, \ldots, x_{i-1}, q, p, x_{i+2}, \ldots, x_{n}\right\rangle,
\end{aligned}
$$

such that different results are produced.

Let $(V, k, \bar{k}, S B)$ be the interim picture when point $p$ is visited according to $\Pi_{1}\left(\mathcal{H}(B)\right.$ ) (i.e., when $q$ is investigated by $\Pi_{2}(\mathcal{H}(B)$ ). (Note that both $p$ and $q$ are not visited when that picture is reached.) Then we need to take the following two points into consideration: 
1. Point $p$ can be altered in the interim picture, but $p$ cannot be after the alteration of $q$.

2. Point $p$ cannot be altered in the interim picture, but $p$ can be after the alteration of $q$.

Since $\mathcal{T}$ is stable, in both cases we arrived at contradictions.

We are ready now to present a sufficient (but not necessary) condition for equivalent transition functions.

Theorem 4. A transition function is equivalent if it is stable.

Proof. If a transition function $\mathcal{T}$ is stable, then it is order-independent by Theorem 3. We need to show that both Algorithms 1 and 2 produce the same result with $\mathcal{T}$. In other words, $P B=S B$ when Algorithms 1 and 2 terminate.

Let $p \in P B$ (i.e., $\mathcal{T}(p, B)=$ false if $p \in B$, or $\mathcal{T}(p, B)=$ true if $p \notin B)$. Since $\mathcal{T}$ is order-independent we can assume that point $p$ is visited first when Algorithm 1 is performed. Then $p$ is evaluated in the initial picture, hence $S B=B$ at that moment. In this case $p \in S B$ since $\mathcal{T}(p, S B)=\mathcal{T}(p, B)$. Thus $p \in S B$ stands for each $p \in P B$. Hence $P B \subseteq S B$.

Conversely, $S B \subseteq P B$ can be seen in the same way. Thus $P B=S B$.

Let us establish a novel sufficient condition for topology-preserving parallel operators.

Theorem 5. A parallel general operator is topology-preserving if its transition function is simple-stable.

Proof. If a transition function $\mathcal{T}$ is simple-stable, then it is order-independent by Theorem 3, and it is equivalent by Theorem 4. Hence the parallel operator with $\mathcal{T}$ and the uniquely specified sequential operator with $\mathcal{T}$ are equivalent. Since $\mathcal{T}$ may alter only simple points, that sequential operator is topology-preserving by Theorem 1. Thus the parallel operator with $\mathcal{T}$ is also topology-preserving.

\section{An Example of Non-Simple-Stable Transition Functions}

Consider the transition function associated with the general operator that was devised by Conway 3. That operator is referred to as "Game of Life", and it is an evolution determined by the initial picture on the (2D) square grid containing points having two possible states, live (black) or dead (white). Two points are called neighbors if they are 8-adjacent [12. At each iteration step the following transitions may happen: a live point with fewer than two live neighbors dies (due to loneliness); a live point with two or three live neighbors stays alive; a live point with more than three live neighbors dies (due to suffering from crowding); a dead point with exactly three live neighbors becomes a live point (otherwise it remains dead).

Figure 1 shows that the transition function of the Conway's operator is not order-independent. Hence it is not stable by Theorem 3. The initial picture 
(see Fig. 1a) contains three amazing objects: they are period 2 oscillators (i.e., if we transform them twice, then we get the initial patterns back). We get three different results when the parallel operator (see Fig. 1 b), and two kinds of sequential operators are performed (see Fig. 11 and Fig. 11d). It is easy to see that a singleton black component is completely deleted by the transition function of the Conway's operator, hence it is not simple-stable (and the "Game of Life" is not topology-preserving).

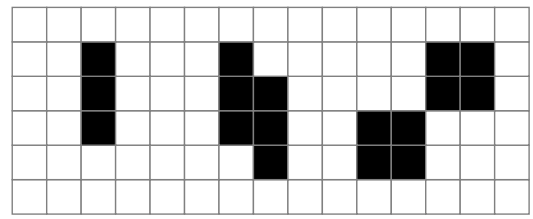

(a) initial picture

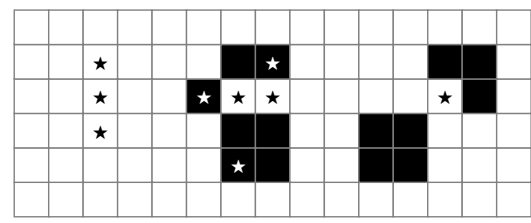

(c) sequential result 1

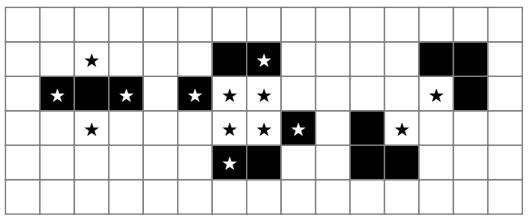

(b) parallel result

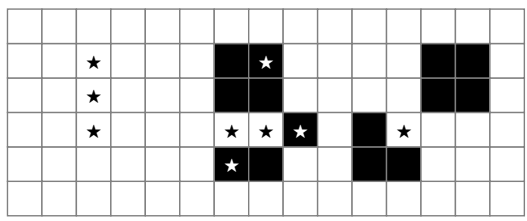

(d) sequential result 2

Fig. 1. Example of a non-simple-stable transition function. The parallel and the two kinds of sequential operators with the transition function of the Conway's operator produce different results (b-d) for the same initial picture (a). The result of the sequential operator with the with the row-by-row traversal (c), and the result with the reverse (i.e., from down-to-up and right-to-left) visiting order (d). Note that these three operators disconnect two black components of the initial picture and both sequential operators completely delete the third black component. Altered points are marked stars.

\section{Examples of Simple-Stable Transition Functions}

In this section a pair of simple-stable transition functions working on the (2D) square grid are presented.

Transition functions $\mathcal{T}_{1}$ and $\mathcal{T}_{2}$ are given by the set of four-four matching templates depicted in Fig. 2. Let us state some properties of the matching templates associated with transition function $\mathcal{T}_{1}$. A point is called alterable if at least one template associated with $\mathcal{T}_{1}$ matches it, and a point is said to be unalterable if it is not alterable. Let $q$ be an alterable point that is matched by a template $T$ associated with $\mathcal{T}_{1}$, and let $p$ be a point in the $3 \times 3$ support of $\mathcal{T}_{1}$ with respect to $q$ (i.e., $p$ is 8 -adjacent to $q$ ). Then the following propositions hold.

Proposition 1. Point $q$ is simple in $(8,4)$ pictures. 

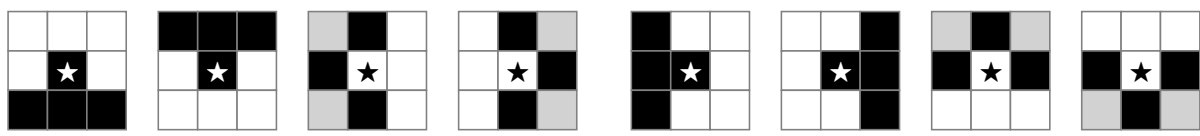

Fig. 2. The first four matching templates are associated with the transition function $\mathcal{S}_{1}$, and the last four templates specify the transition function $\mathcal{S}_{2}$. A point is matched by a template if each black position matches a black point and each white element matches a white point. The template position depicted in grey matches either a black or a white point. The central element of a template is marked by " $\star$ ".

It is easy to see that conditions of Theorem 2 hold for each alterable point.

Proposition 2. If $p$ is matched by a black or a white position in $T$, then $p$ is unalterable and remains unalterable after $q$ is altered.

Proposition 3. If $p$ is matched by a position depicted in grey $T$, then the alterability of $p$ remains unchanged after $q$ is altered.

Propositions 2 and 3 are obvious by careful examination of the templates associated with $\mathcal{T}_{1}$. The following theorem is an easy consequence of Propositions $1-3$.

Theorem 6. Transition function $\mathcal{T}_{1}$ is simple-stable.

Since the four matching templates associated with $\mathcal{S}_{2}$ can be get by rotating the templates of $\mathcal{T}_{2}$ (where the rotation angle is $90^{\circ}$ ), $\mathcal{T}_{2}$ is also simple-stable.

Figure 3 is to illustrate a parallel (or sequential) algorithm for contour smoothing. It is composed of the operator with transition function $\mathcal{T}_{1}$ followed by the operator with transition function $\mathcal{T}_{2}$. Note that this algorithm is topologypreserving by Theorems 5 and 6 .
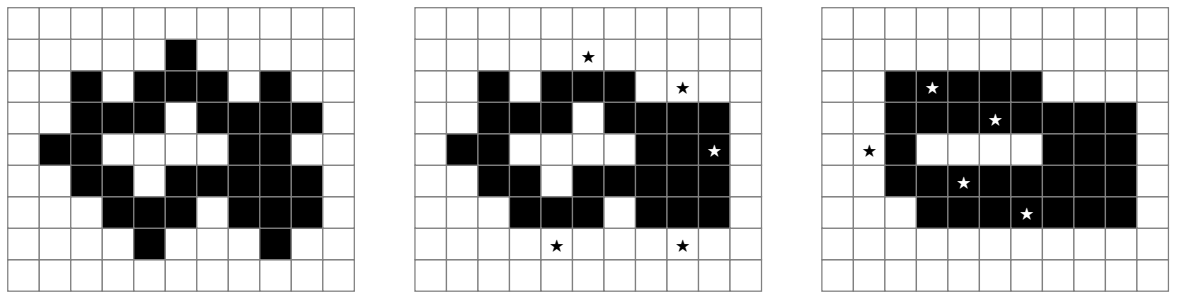

Fig. 3. Example of the 2-phase smoothing algorithm. The result of the operator with transition function $\mathcal{T}_{1}$ (middle) for the initial picture (left). The process is completed by the operator with $\mathcal{T}_{2}$ (right). Altered points are marked stars.

Acknowledgements. This work was supported by the European Union and co-funded by the European Social Fund. Project title: "Telemedicine-focused research activities on the field of Mathematics, Informatics and Medical sciences." Project number: TÁMOP-4.2.2.A-11/1/KONV-2012-0073. 


\section{References}

1. Couprie, M., Bertrand, G.: New characterizations of simple points in 2D, 3D, and 4D discrete spaces. IEEE Transactions on Pattern Analysis and Machine Intelligence 31, 637-648 (2009)

2. Faisan, S., Passat, N., Noblet, V., Chabrier, R., Meyer, C.: Topology preserving warping of 3-D binary images according to continuous one-to-one mappings. IEEE Transactions on Image Processing 20, 2135-2145 (2011)

3. Gardner, M.: Mathematical Games: The fantastic combinations of John Conway's new solitaire game "life". Scientific American 223, 120-123 (1970)

4. Hall, R.W.: Parallel connectivity-preserving thinning algorithms. In: Kong, T.Y., Rosenfeld, A. (eds.) Topological Algorithms for Digital Image Processing, pp. 145179. Elsevier Science B.V. (1996)

5. Hall, R.W., Kong, T.Y., Rosenfeld, A.: Shrinking binary images. In: Kong, T.Y., Rosenfeld, A. (eds.) Topological Algorithms for Digital Image Processing, pp. 3198. Elsevier Science B.V. (1996)

6. Han, X., Xu, C., Prince, J.L.: A topology preserving level set method for geometric deformable models. IEEE Transactions on Pattern Analysis and Machine Intelligence 25, 755-768 (2013)

7. Kardos, P., Palágyi, K.: On topology preservation in triangular, square, and hexagonal grids. In: Proc. 8th Int. Symposium on Image and Signal Processing and Analysis, ISPA 2013, pp. 782-787 (2013)

8. Kardos, P., Palágyi, K.: Sufficient conditions for general 2D operators to preserve topology. In: Barneva, R.P., Brimkov, V.E., Šlapal, J. (eds.) IWCIA 2014. LNCS, vol. 8466, pp. 101-112. Springer, Heidelberg (2014)

9. Knuth, D.E.: Art of Computer Programming: Sorting and Searching, 2nd edn., vol. 3. Addison-Wesley Professional (1998)

10. Kong, T.Y.: On topology preservation in 2-d and 3-d thinning. Int. Journal of Pattern Recognition and Artificial Intelligence 9, 813-844 (1995)

11. Kong, T.Y.: Topology-preserving deletion of 1's from 2-, 3- and 4-dimensional binary images. In: Ahronovitz, E. (ed.) DGCI 1997. LNCS, vol. 1347, pp. 1-18. Springer, Heidelberg (1997)

12. Kong, T.Y., Rosenfeld, A.: Digital topology: Introduction and survey. Computer Vision, Graphics, and Image Processing 48, 357-393 (1989)

13. Németh, G., Palágyi, K.: Topology preserving parallel thinning algorithms. International Journal of Imaging Systems and Technology 23, 37-44 (2011)

14. Palágyi, K.: Deletion Rules for Equivalent Sequential and Parallel Reductions. In: Ruiz-Shulcloper, J., Sanniti di Baja, G. (eds.) CIARP 2013, Part I. LNCS, vol. 8258, pp. 17-24. Springer, Heidelberg (2013)

15. Ranwez, V., Soille, P.: Order independent homotopic thinning for binary and grey tone anchored skeletons. Pattern Recognition Letters 23, 687-702 (2002)

16. Serra, J.: Image Analysis and Mathematical Morphology. Academic Press (1982) 